Itinéraires Itinéraires

Littérature, textes, cultures

2014-3 | 2015

Le polar en Europe : réécritures du genre

\title{
Le Nouveau Roman et le roman policier : éloge ou parodie?
}

\section{Simon Kemp}

\section{(2) OpenEdition}

\section{Journals}

Édition électronique

URL : http://journals.openedition.org/itineraires/2579

DOI : $10.4000 /$ itineraires.2579

ISSN : 2427-920X

Éditeur

Pléiade

Référence électronique

Simon Kemp, «Le Nouveau Roman et le roman policier : éloge ou parodie ? », Itinéraires [En ligne], 2014-3 | 2015, mis en ligne le 25 septembre 2015, consulté le 19 avril 2019. URL : http://

journals.openedition.org/itineraires/2579; DOI : 10.4000/itineraires.2579

Ce document a été généré automatiquement le 19 avril 2019

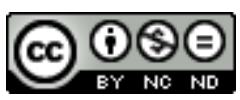

Itinéraires est mis à disposition selon les termes de la licence Creative Commons Attribution - Pas d'Utilisation Commerciale - Pas de Modification 4.0 International. 


\title{
Le Nouveau Roman et le roman policier : éloge ou parodie?
}

\author{
Simon Kemp
}

\section{Introduction}

1 Le genre populaire du roman policier offrait un attrait apparemment irrésistible pour un certain nombre des Nouveaux Romanciers des années 1950 et 1960. Nombreux sont les détectives, les crimes, les mystères à élucider et les enquêtes à mener dans des romans de Robert Pinget, de Michel Butor, et surtout d'Alain Robbe-Grillet. Citons notamment L'Emploi du temps de Butor (1956), L'Inquisitoire de Pinget (1962), et de Robbe-Grillet, son premier roman publié, Les Gommes (1953), son livre suivant, Le Voyeur (1955), et les mélanges de stéréotypes tirés de romans noirs sensationnels et pornographiques qui constituent ses «Nouveaux Nouveaux Romans 》 des années 1960 et 1970, comme La Maison de rendez-vous (1965) ou Projet pour une révolution à New York (1970). Chez d'autres romanciers du groupe, on discerne aussi quelques éléments tirés du genre policier dans Le Vent (1957) de Claude Simon, ou La Mise en scène (1958) de Claude Ollier. Même Nathalie Sarraute, qui est peut-être la seule représentante des Nouveaux Romanciers chez qui on ne distingue pas d'influence perceptible, se voit louée par Sartre pour avoir écrit, avec Portrait d'un inconnu (1948), « un anti-roman qui se lit comme un roman policier » (Sartre [1948] 1996: 35). L'intertextualité qui foisonne entre le roman policier et le roman littéraire a été souvent remarquée, notamment par Marc Lits dans Le Roman policier, introduction à la théorie et à l'histoire d'un genre (1999), par exemple, mais les liens entre Nouveau Roman et roman policier font de leur rapport un cas particulier, comme Ludovic Janvier l'a noté dans l'une des premières études sérieuses du Nouveau Roman, Une parole exigeante ${ }^{(1964)}$. Moins nombreux dans ces Nouveaux Romans policiers, il faut l'admettre, sont les malfaiteurs arrêtés, les mystères éclaircis ou les enquêtes bien bouclées. Si les thèmes, les personnages et les structures de l'intrigue du roman policier sont souvent présents dans ces romans d'avant-garde, les plaisirs simples de la lecture d'un bon polar - suivre une intrigue complexe, résoudre une énigme difficile en parallèle avec le détective du roman, ou voir l'ordre restitué dans une situation marquée par le chaos 
moral et épistémologique - semblent plutôt manquer. Dans le Nouveau Roman, le détective échoue, le criminel s'échappe et, le mystère, quant à lui, reste le plus souvent sans résolution, se perdant en détails innombrables ou s'évanouissant dans le doute et le non-événement. Quelle est donc la nature du rapport entre les romans expérimentaux d'après-guerre et le genre dont ils tirent leurs influences? Peut-on parler d'éloge ou d'hommage lorsque les intentions du genre original sont tellement déformées dans leur reflet littéraire ? Est-ce plutôt un détournement du polar dans le but de créer une parodie hostile, peut-être pour se moquer de la naïveté du genre et de ses lecteurs ? Est-il possible d'être hommage et parodie à la fois ?

\section{L'imitation du genre}

2 Selon Gérard Genette (1982: 31-32), les relations parasitiques d'un «hypertexte " à son " hypotexte » antérieur s'agencent sur un mode ludique, satirique ou sérieux, et mettent en valeur ou l'imitation ou la transformation du modèle. Des deux modes «ludiques » de l'hypertextualité dans la typologie de Genette, le pastiche accentue les traits stylistiques de l'original à travers une imitation, tandis que la parodie insiste plutôt sur sa transformation. Tout en nous gardant bien de situer le Nouveau Roman dans l'une ou l'autre catégorie - notre objectif n'est pas de "caser» ces textes complexes dans un système trop simpliste - commençons par examiner les éléments d'imitation et de transformation dans ces polars expérimentaux. Chez Robbe-Grillet, l'imitation la plus étendue du genre conventionnel se trouve dans Les Gommes. Dans ce roman, le récit commence par un crime - l'assassinat raté de Daniel Dupont par un membre d'un groupe terroriste - puis le lecteur suit l'enquête du policier Wallas à travers l'inspection des lieux, la recherche de témoins et l'interrogation des suspects, jusqu'à ce qu'il découvre la vérité de l'affaire dans un dénouement violent. (Laissons de côté pour le moment le fait que cette violence éclate lorsque le détective abat la « victime " par erreur, persuadé que la personne qui se trouve devant lui est coupable d'un attentat qu'il croit encore, à ce moment-là, réussi.) L'ambiance est aussi d'un noir authentique : le détective cynique et las $\mathrm{du}$ monde s'accorde parfaitement au décor gris d'une ville industrielle peu accueillante, sur laquelle pèse une atmosphère de menace mal définie. Bien que RobbeGrillet n'aille pas jusqu'à recopier des pages entières de Simenon, comme le fera plus tard Jacques Roubaud dans La Belle Hortense (1985), il s'approprie à volonté le style parfois galvaudé du roman noir américain ou européen du milieu du siècle et les situations transformées en clichés par les thrillers d'Hollywood ${ }^{1}$ :

Wallas arrive à cette petite porte vitrée dont lui a parlé le commissaire. Il frappe au carreau, de son index replié. La vieille gouvernante ayant de nouveau disparu, il essaye de tourner la poignée; la porte n'est pas fermée. Il pousse le battant, qui grince sur ses gonds, comme dans une maison abandonnée - hantée peut-être - où chaque geste déchaîne un vol de hiboux et de chauves-souris. Mais une fois le vantail refermé, aucun froissement d'ailes ne trouble le silence. Wallas fait en hésitant quelques pas; ses yeux qui s'habituent au demi-jour glissent sur les boiseries, les moulures compliquées, la colonne de cuivre qui trône au seuil de l'escalier, les tapis, tout ce qui faisait, au début du siècle, l'ornement d'une demeure bourgeoise.

Wallas sursaute en entendant tout à coup la voix de Mme Smite qui l'appelle au bout du couloir. Il se retourne et aperçoit la silhouette qui se détache sur le vitrage de la petite porte. L'impression l'effleure, un instant, qu'il vient de se laisser prendre dans un piège. (Robbe-Grillet 1953 : 90) 
Ces éléments qui sont familiers aux lecteurs du genre authentique deviennent encore plus évidents dans ses « Nouveaux Nouveaux Romans » des années 1960 et 1970. Robbe-Grillet, se désintéressant progressivement de la psychologie de ses personnages et de la cohérence du récit, commence à rompre les liens entre son univers fictif et le monde réel et s'ancre d'autant plus dans les tropes de la fiction populaire. La Maison de rendez-vous (1965) et Projet pour une révolution à New York (1970) puisent leurs personnages et situations dans la littérature à sensation d'écrivains comme Sax Rohmer, auteur d'une série de romans de gare orientalistes sur le génie criminel Fu Manchu, ou les romans noirs américains de Mickey Spillane, dont la représentation de la violence et de la sexualité frôle la pornographie. Trafic d'opium et prostituées asiatiques dans l'un et tueur en série sadique entre les gratte-ciel dans l'autre, ces romans rassemblent et recombinent les clichés d'une fiction choisie pour son statut d'antithèse du roman littéraire.

4 L'imitation des styles, des thèmes et des structures du roman policier apparait clairement chez d'autres Nouveaux Romanciers. Chez Pinget, dans L'Inquisitoire, nous assistons à la disparition mystérieuse d'un employé du château de Broy et à l'interrogatoire d'un autre employé, témoin possible de l'événement, dans un dialogue qui rappelle ceux du roman noir: "Croyez-vous que nous vous interrogions pour le plaisir, dites tout ce que vous avez vu », exige l'interrogateur implacable (Pinget 1962 : 273). Chez Butor, nous n'avons qu'un détective amateur, qui recherche le coupable dans une affaire de tentative de meurtre présumée, mais les liens au genre populaire sont renforcés dans L'Emploi du temps par le fait que la victime présumée est auteur de romans policiers, dont l'un, Le Meurtre de Bleston, contient peut-être l'indice essentiel dans l'affaire. Accompagnant ce polar en abyme (dont, il faut le dire, peu de détails nous sont dévoilés) dans le roman de Butor, le roman nous offre un discours sur l'art du roman policier par son auteur, un certain M. Burton. Ce discours se centre sur la structure du récit dans le roman policier, et souligne implicitement les parallèles entre l'écriture de Burton et celle de Butor. Burton nous explique :

Dans le roman policier, le récit est fait à contre-courant, puisqu'il commence par le crime, aboutissement de tous les drames que le détective doit retrouver peu à peu [...]; le récit explore peu à peu des événements antérieurs à celui par lequel il commence, ce qui peut déconcerter certains, mais qui est tout à fait naturel [...] puisque dans la réalité, trop souvent, c'est seulement lorsque l'explosion du malheur est venue troubler notre vie que, réveillés, nous recherchons ses origines. (Butor 1956 : 171)

Effectivement, le narrateur du roman de Butor, Jacques Revel, construit son propre récit à contre-courant. Hanté par le malaise qui semble se répandre sur sa vie depuis son arrivée à Bleston, puis par les circonstances suspectes de «l'accident » de Burton, il revient en pensée aux premiers mois de son séjour, à la recherche de la solution à ces deux mystères. Ce n'est que plus tard qu'il se rendra compte que sa procédure imite celle de l'écrivain de polars, et que le «journal » rétrospectif qu'il écrit à partir de ses exercices de mémoire présente une structure similaire à celle des romans policiers. La lecture de polars par les personnages du « Nouveau Roman policier» ne se limite pas au roman de Butor. Dans Projet pour une révolution à New York, Robbe-Grillet joue avec les niveaux de fiction lorsque la description de l'illustration de couverture salace et macabre d'un roman policier glisse imperceptiblement vers la description d'une scène qui existe réellement dans la diégèse du roman. Comme chez Butor, la structure du récit se voit également impliquée par ce lien entre roman d'avant-garde et roman de genre. Le narrateur du roman de Robbe- 
Grillet explique qu'il trouve souvent les polars de son amante qui traînent un peu partout chez lui :

Ce qui m'a toujours fait supposer que Laura lisait tous ces livres en même temps et qu'elle en mélangeait ainsi de pièce en pièce, selon ses propres déplacements, les péripéties policières savamment calculées par l'auteur, modifiant donc sans cesse l'ordonnance de chaque volume, sautant de surcroît cent fois par jour d'un ouvrage à l'autre, ne craignant pas de revenir à plusieurs reprises sur le même passage pourtant dépourvu de tout intérêt visible, alors qu'elle délaisse au contraire totalement le chapitre essentiel qui contient le nœud de l'enquête, et par conséquent sa signification à l'ensemble de l'intrigue; et cela d'autant plus que, beaucoup de ces brochures de fabrication médiocre ayant mal résisté à la négligence parfois brutale d'un tel mode de lecture, elles ont perdu, au cours des mois, un coin de feuillet, une page entière çà et là ou même deux ou trois cahiers d'un seul coup. (Robbe-Grillet $1970: 85$ )

Robbe-Grillet propose ironiquement au lecteur de comparer l'expérience de la lecture de Projet pour une révolution à un collage de fragments tirés de divers polars, découpés et rassemblés en désordre. L'incohérence voulue du roman, qui saute d'une situation à une autre, mélange les personnages, ou s'arrête brusquement au milieu de l'action pour répéter le même passage mais avec des variations, ne manque pas de nous donner parfois cette impression. Une telle réflexivité fait partie du genre policier depuis ses débuts au XIX ${ }^{e}$ siècle: Sherlock Holmes, par exemple, avait l'habitude de critiquer le style et le contenu des récits censément publiés par Watson ${ }^{2}$. Dans Projet pour une révolution aussi, l'interrogation du narrateur par la police comporte des critiques de sa déposition en des termes qui imitent ceux d'un lecteur hostile ou d'un critique littéraire à la recherche de contradictions dans l'histoire. Cependant, en contraste avec L'Emploi du temps, le texte en abyme de Projet pour une révolution ne sert pas à insister sur les similarités entre la structure du roman et celle du polar. Chez Robbe-Grillet, c'est l'extrême cohérence même de l'intrigue du roman policier, et l'importance capitale de son dénouement, qui rendent singulières les habitudes de lecture de Laura et insistent par conséquent sur le sabotage pratiqué par l'auteur sur les conventions du genre ${ }^{3}$. Nous passons de l'imitation à la transformation du modèle.

\section{La transformation du genre}

7 L'hypotexte du polar sert à Robbe-Grillet de "générateur » narratif, et le roman policier n'est pas pour lui la seule source de ces générateurs :

Mes thèmes générateurs sont choisis de plus en plus [...] dans l'imagerie populaire contemporaine (« populaire » au sens large car la circulation de ces images entre les classes est totale, dans nos sociétés dites avancées) : couvertures illustrées des romans qu'on vend dans les gares, affiches géantes. Revues pornographiques des sex-shops, publicités vernies des magazines de mode, figures peintes à plat des bandes dessinées. (Robbe-Grillet $1971: 161$ )

8 L'attitude du romancier envers son matériel est loin d'être un éloge de la créativité de la culture populaire. Il se montre esthétiquement et politiquement hostile :

Vous voyez qu'il n'y a dans la reprise de ces thèmes (qui me servent de générateurs) aucune soumission aux codes de la société en place - pas plus au code des valeurs qu'au code narratif - mais au contraire un travail de déconstruction sur les éléments découpés dans le code. (Robbe-Grillet 1971 : 160) 

prétendent que les derniers romans de Robbe-Grillet ne font que reproduire les tropes de la pire pornographie sadique et misogyne. Mais il y a aussi dans cette déclaration un aspect important de la relation entre les Nouveaux Romanciers et le roman policier. Là où Butor s'inspire de la complexité chronologique de la narration du polar (selon l'analyse de George Burton) pour créer dans L'Emploi du temps une sorte de canon musical de niveaux temporels entrelacés ${ }^{4}$, Robbe-Grillet s'inspire de la cohérence de l'intrigue du roman policier - son besoin que tout concorde - pour créer un roman qui fait sentir son incohérence au maximum. La relation entre le polar et le Nouveau Roman se base sur deux éléments contradictoires : le parallélisme et l'opposition absolue.

Ce paradoxe se révèle le plus clairement dans le dénouement de ces textes. Le Nouveau Roman emprunte au polar sa fixation sur la fin de l'histoire, le moment de révélation lorsque la vérité du crime et l'identité du malfaiteur se laissent voir. Le détective présente les preuves au lecteur, et dans la version classique, aux suspects rassemblés aussi; il éclaircit les tentatives de brouiller la piste et écarte les détails sans importance de l'histoire. Petit à petit il reconstruit pour nous l'enchaînement de raisonnements qui l'a amené du mystère initial du crime à sa résolution indubitable. Implicitement, l'auteur nous invite à vérifier et à revérifier son calcul. Si la solution offerte nous paraît inattendue, mais que tout se tient quand même dans son explication, le polar a réussi. Butor met ce procédé typique du genre en contraste avec l'effet qu'il a voulu produire par le dénouement de L'Emploi du temps :

Le roman policier est un genre populaire, commercial, et il est fait pour qu'on ne s'en souvienne plus. Le roman policier est fait pour que nous le lisions en une soirée. Et une fois que nous l'avons lu, les choses se referment. Il y a cet aspect final qui explique tout. Le coupable est puni. Nous pouvons dormir tranquilles. [...] Je veux empêcher les gens de dormir. [...] Je veux faire le contraire de ce que fait le roman policier dans son utilisation habituelle. Le roman policier est fait pour que les gens dorment. C'est un médicament, si vous voulez. C'est un calmant très efficace, très bien fait, qui fonctionne très bien. En étudiant la façon dont il fonctionne, on peut essayer de faire des drogues qui aient un autre effet. (Butor et Rice-Sayre 1977 : 109-111)

Dans le roman de Butor, l'enquête de Revel sur la tentative de meurtre dont son ami a supposément été victime se perd dans les détails accumulés. Le moindre incident doté d'une signification possible prend de l'ampleur avec chaque nouvelle nuance retrouvée par Revel dans sa mémoire, puis se dédouble avec chaque nouveau récit qu'il en fait dans son journal, où il relit et remanie d'une manière obsessionnelle l'histoire de l'accident de Burton. Lorsqu'il trouve un suspect principal dans l'affaire, son ancien ami James, il ne s'aperçoit pas que les activités « louches » de ce dernier sont motivées moins par le besoin de cacher un crime affreux que par le désir de dissimuler sa relation amoureuse avec la femme dont Revel est, lui aussi, amoureux. Revel ne se rend même pas compte que les déductions froides et rationnelles par lesquelles il arrive à la conclusion de la culpabilité de James ne sont que les erreurs d'un jaloux qui rationalise sa haine envers son rival en masquant son émotion d'un vernis de logique. Ce n'est qu'à la fin du roman qu'il sera enfin capable de s'avouer la vérité qui réside derrière ses soupçons :

Malgré moi mais par moi, pour ma transformation en ce fantôme que je suis devenu, se sont rejoints cette Ann qui m'avait aimé [...] et ce James, dont je suis arrivé à me persuader qu'il était coupable d'une tentative de meurtre. (Butor 1956 : 341) 
Dans cet « arrivé à me persuader » on perçoit toute la fragilité de la raison objective, qui se voit si facilement corrompue par les préjugés personnels.

Ce n'est pas que l'effet déformant des sentiments qui empêche les enquêteurs d'y voir clair dans leur mystère. De différentes manières, tous ces Nouveaux Romans policiers méditent sur l'impossibilité de faire revivre le passé. Chez Pinget, le héros de L'Inquisitoire répond d'une façon pointilleuse à toutes les questions des interrogateurs, même à celles qui n'ont aucun lien concevable avec la disparition du secrétaire. Après des digressions sans fin sur l'agencement du château et de ses environs, sur son propre passé et celui de toutes ses connaissances, il ne perd patience qu'au dénouement du roman, lorsque l'interrogation revient finalement aux motivations éventuelles de la fuite du secrétaire :

\begin{abstract}
Lorsque vous y repensez à tête reposée au café par exemple que soupçonnez-vous qui ait pu provoquer la décision du secrétaire?

Si vous croyez que je pense à ça vous vous trompez il y a beau temps que je ne me tracasse plus sur les raisons de ce qui nous arrive on sait ce qu'on perd chaque jour et ça suffit, la mémoire un temps j'aurais donné dix ans de ma vie pour la ravoir et maintenant même plus elle vous laisse comme elle vous a trouvé on en sait de moins en moins, le tas de choses molles on essaie d'abord d'y repêcher des bribes ensuite plus on prend ce qui vient et on rêvasse sur des souvenirs tout faux ce qu'on aurait voulu et qu'on n'a pas eu, ça on s'en souvient longtemps et un temps vient où on ne s'en souvient plus et c'est la fin on est bon pour l'autre côté, les morts voyezvous ils ne se souviennent de rien mais est-ce que ce n'est pas normal vous qui voulez du normal, est-ce que ça ne l'est pas dites-moi qu'ils ne se souviennent plus quand on pense nous déjà tout ce fatras de jours sans que ni tête le travail le repos les projets la maison à la campagne la famille pour ce que ça donne et pour ce qu'on en a fait on l'oublie aussi.

Répondez, quels étaient donc vos soupçons à l'époque ? (Pinget 1962 : 473-474)
\end{abstract}

Malgré leurs tentatives incessantes, les interrogateurs ne réussissent pas à en tirer davantage du vieux domestique. En substituant ce discours mélancolique à la résolution du mystère qui conclurait normalement un vrai roman policier, le roman de Pinget se révèle aux dernières pages avoir été depuis le début, non pas la recherche d'une vérité banale comme il le prétendait, mais une étude de la mémoire, de ses défaillances, de ses particularités, de sa contenance parfois ahurissante, et surtout de la douleur qui découle de sa capacité à nous relier à un passé à jamais perdu.

Chez d'autres, l'échec de l'enquête représente quelque chose de moins émotif, et de plus philosophique. L'absence de solution aux mystères chez Robbe-Grillet prend une signification d'ordre épistémologique. Revenant sur sa carrière d'écrivain dans le premier volet de son autobiographie "romanesque ", Robbe-Grillet met en valeur un thème qui sous-tend son œuvre en général et plus particulièrement ses romans d'ambiance policière :

Mettre les choses en ordre. Définitivement ! La vieille obsession naïve reparait çà et là, ironique, insistante, désespérée, à travers tout mon travail romanesque, dont le héros multiforme récapitule sans relâche son emploi du temps à la charpente trop fragile, compte et recompte ses bananiers mouvants, règle méticuleusement des supplices, ou reprend inlassablement le même épisode (espérant chaque fois en venir à bout de façon logique et rationnelle), la relation par exemple de ce qu'il a exactement vu et fait, à la Villa Bleue, le soir en question. (Robbe-Grillet 1984:59)

C'est dans La Maison de rendez-vous qu'il est question de la soirée à la Villa Bleue, et l'emploi du temps à la charpente fragile se trouve dans Le Voyeur. Dans les deux romans, le narrateur, mi-criminel mi-enquêteur, essaie de fixer les événements de la période du crime, mais se perd toujours en conjectures, en ouï-dire ou en fantaisies. De même, dans 
L'Emploi du temps, Revel remplit les dernières pages de son journal de regrets quant au fait que son récit sera « inévitablement insuffisant, inévitablement lacunaire " (Butor 1956: 381). Comme les enquêteurs de Robbe-Grillet, il s'est perdu dans un labyrinthe, dans lequel les faits objectifs et les liens causaux qui les relient restent péniblement hors de sa portée. Se souvenant à la fin du roman de la conversation clé avec Burton qui lui a fait croire pour la première fois à un complot criminel, il s'émerveille de la profusion épistémologique qui en a résulté :

Sur cette conversation du milieu de l'hiver [...], quelle végétation s'est développée soutenant cet instant présent, cet observatoire d'où je la repère, quelle végétation d'événements et de pensées, d'oublis, de réflexions, de tentatives, immense échafaudage de branches bourgeonnantes, se ramifiant, se rencontrant, se faisant ombre, se traversant, se réunissant, se faisant guerre, immense échafaudage de poutres vivantes que toutes les pages de cette semaine explorent, reconnaissant à des niveaux intermédiaires toute une série de relais ou d'échelons sur lesquels mon effort de mémoire ce soir prenait appui pour parvenir jusqu'à ce sol d'antan? (Butor 1956 : 381)

Dans ces derniers exemples, ce ne sont plus les faiblesses de la mémoire ou les déformations causées par la jalousie, éléments qui sont d'ailleurs tout à fait admissibles dans le genre policier lui-même, qui entrent en jeu pour empêcher l'accès à la vérité. Le problème réside plutôt dans la nature même de la réalité et dans la capacité du cerveau humain à la comprendre. Reprenant la vision des existentialistes de la génération précédente, les Nouveaux Romanciers représentent dans leurs fictions la quête de l'ordre et de la raison dans un univers résolument incompréhensible. C'est un univers postmoderne, dans lequel la vérité et le mensonge ne sont que relatifs, où la causalité n'est qu'une histoire inventée pour lier les événements antérieurs à ceux qui les suivent, et où les faits significatifs s'estompent en gros plan derrière des myriades de détails qui prolifèrent comme dans l'agrandissement d'une image fractale. Le pessimisme épistémologique des Nouveaux Romanciers, face à un monde qui reste étranger à nos efforts pour le comprendre et le maîtriser, cherche son expression dans une forme littéraire qui puisse le mettre en valeur. Le roman policier leur convient à merveille à cause de deux éléments essentiels de la forme: premièrement, le genre met en scène l'affrontement entre la raison humaine et la réalité qu'elle essaie de décrypter, affrontement que les Nouveaux Romanciers imitent dans leurs propres ouvrages; deuxièmement, le roman policier les attire à cause de sa foi optimiste que l'observation et la déduction réussiront à rendre compréhensible cette réalité, perspective à laquelle ils s'opposent absolument.

\section{Conclusion : de victimes et de complices}

Malgré les paroles peu flatteuses prononcées par Butor et Robbe-Grillet à propos du polar, il serait erroné de voir dans leurs œuvres une attaque contre le genre. Certes, l'optimisme épistémologique du roman policier serait naïf dans le monde réel, où chaque mystère ne trouve pas nécessairement sa solution. Mais les lecteurs de polars, un groupe qui inclut bien sûr les Nouveaux Romanciers, savent parfaitement bien distinguer le genre de la vraie vie. Personne ne croit que la conception de la raison et de la vérité qui est présentée dans le roman policier reflète fidèlement l'efficacité de la raison ou l'accessibilité de la vérité dans notre réalité moins cohérente. Personne n'a besoin d'un Nouveau Roman pour prendre conscience de ce fait, et les Nouveaux Romanciers, eux aussi, en sont 
parfaitement conscients : ils n'écrivent pas pour éclairer un lecteur tellement plongé dans l'ignorance qu'il croit habiter le monde de Georges Simenon. Écrire pour démontrer au lecteur que les criminels échappent parfois aux détectives serait ridicule, et il est clair que ces romanciers visent quelque chose de plus ambitieux. En effet, le roman policier n'est pas vraiment la cible de leurs critiques : comme nous l'avons vu, le genre n'est que le véhicule de leurs méditations philosophiques sur la raison et la vérité, ou de leurs pensées mélancoliques sur la mémoire et le passé.

Si les Nouveaux Romanciers gardent tout de même leurs distances avec le genre, et se moquent de ses défaillances plus qu'ils ne célèbrent son esprit d'invention, la génération suivante effectuera en quelque sorte un rapprochement. Dans «53 jours» (1989) de Georges Perec ou Cherokee (1983) de Jean Echenoz, on voit la même appropriation des thèmes et structures du polar au service de buts littéraires beaucoup plus larges que la critique d'un genre populaire. Cependant, le ton de leurs romans exprime un véritable enthousiasme pour le genre populaire, et l'amateur de polars se voit accueilli chaleureusement par un écrivain qui partage son plaisir. En même temps, dans le genre lui-même, des écrivains comme Sébastien Japrisot empruntent les jeux déconstructifs du Nouveau Roman pour examiner la nature de l'identité ou de la vérité, tout en restant dans la forme reconnaissable du roman policier. La coopération entre fiction littéraire et genre populaire est devenue plus évidente, peut-être, dans les décennies qui sont venues après l'apogée du Nouveau Roman. Mais même dans les années 1950, quand Robbe-Grillet et Butor publiaient leurs premiers Nouveaux Romans policiers, le genre était loin d'être simplement l'objet de leur mépris. Leur attitude est plus proche de celle que prête Mikhail Bakhtine (1981: 413) à Cervantes envers les romans de chevalerie : en équilibre délicat entre la critique et le soutien. Si ces romanciers d'avant-garde n'écrivent pas exactement l'éloge du polar, ils n'en écrivent certainement pas la parodie : ils trouvent plutôt dans le genre populaire un collaborateur, parfois réticent, parfois involontaire, dans leur mission de repenser notre conception de ce que nous connaissons, et de ce qui peut être connu, du monde qui nous entoure et du passé qui persiste dans nos mémoires.

\section{BIBLIOGRAPHIE}

Bakhtine, Mikhail, 1981, The Dialogic Imagination, ed. Michael Holquist, trad. par Carol Emerson et Michael Holquist, Austin, University of Texas Press.

Butor, Michel, 1956, L'Emploi du temps, Paris, Minuit.

-, 1993, Improvisations sur Michel Butor : l'écriture en transformation, Paris, La Différence.

Butor, Michel et Rice-Sayre, Laura, 1977, « Le roman policier et le Nouveau Roman : entretien avec Michel Butor », French-American Review, nº 1, p. 101-114.

Conan Doyle, Arthur, 1981, The Penguin Complete Sherlock Holmes, Londres, Penguin.

Genette, Gérard, 1982, Palimpsestes : la littérature au second degré, Paris, Seuil. 
Gorrara, Claire, 2003, The Roman Noir in Post-War French Culture: Dark Fictions, Oxford, Oxford University Press.

Janvier, Ludovic, 1964, Une parole exigeante : le nouveau roman, Paris, Minuit.

Lits, Marc, 1999, Le Roman policier : introduction à la théorie et à l'histoire d'un genre littéraire, Liège, Éditions du Céfal.

Pinget, Robert, 1962, L'Inquisitoire, Paris, Minuit.

Robbe-Grillet, Alain, 1953, Les Gommes, Paris, Minuit.

-, 1970, Projet pour une révolution à New York, Paris, Minuit.

-, 1971, «Sur le choix des générateurs », dans J. Ricardou et F. van Rossum-Guyon (dir.), Nouveau

Roman : hier, aujourd'hui, t. II, Pratiques, Paris, UGE, coll. « 10/18 », p. 157-162.

,- 1984, Le miroir qui revient, Paris, Gallimard.

Sartre, Jean-Paul, [1947] 1996, «Préface à Portrait d'un inconnu », dans N. Sarraute, CEuvres

complètes, éd. Jean-Yves Tadié, Paris, Gallimard, p. 35-39.

Todorov, Tzvetan, 1971, « Typologie du roman policier », dans Poétique de la prose, Paris, Seuil, p. 55-65.

\section{NOTES}

1. Le roman noir français, dont les écrivains notables incluent Léo Malet et Jean-Patrick Manchette, adopte et transforme les traits du roman à suspense américain de Dashiell Hammett ou de Raymond Chandler, en l'adaptant à un milieu européen. Comme Tzvetan Todorov le remarque dans son « Typologie du roman policier » (1971), la « fusion » du récit de l'enquête avec le récit du crime, mettant ainsi l'accent sur les aventures d'un détective menacé par des dangers inconnus, caractérise le genre. Claire Gorrara nous offre l'analyse du genre français dans The Roman Noir in Post-War French Culture: Dark Fictions (2003).

2. Pour ne citer qu'un seul exemple, dans "The Adventure of the Abbey Grange " [1904], Holmes accuse Watson de sensationnalisme et de manque de précision: "You slur over work of the utmost finesse and delicacy, in order to dwell upon sensational details which may excite, but cannot possibly instruct, the reader.» («Vous expédiez du travail d'une finesse et d'une délicatesse extrêmes pour vous attarder sur des détails sensationnels qui peuvent exciter le lecteur, mais ne peuvent absolument pas l'instruire. ») Arthur Conan Doyle ([1904] 1981 : 636).

3. Il faut bien reconnaître que si l'intrigue de L'Emploi du temps ne rivalise pas avec l'incohérence voulue de celle de Robbe-Grillet, elle n'aboutit pas non plus à un dénouement résolu. Le manque de temps du narrateur et l'embrouillement de son jugement par la jalousie font que son récit reste sans conclusion satisfaisante.

4. La métaphore du canon pour décrire la structure de L'Emploi du temps vient de l'auteur, qui appelle son roman « une structure musicale à cinq voix dont deux rétrogrades » (Michel Butor $1993: 84)$. 


\section{RÉSUMÉS}

Le roman policier était un objet de fascination pour les Nouveaux Romanciers. La liste des ouvrages qui s'approprient les thèmes, les personnages ou la structure narrative du polar est longue, et comprend des textes de Robbe-Grillet, Simon, Pinget et Butor. La forme fixe du polar, et le thème dominant de la restitution de l'ordre à l'aide de la raison et de la vérité, se sont avérés irrésistibles pour un mouvement littéraire qui visait à remettre en question toute présupposition esthétique ou épistémologique de la fiction romanesque. Comment caractériser ce rapport d'imitation entre le Nouveau Roman et le roman policier, qui ne semble pas être une parodie hostile, mais qui n'est pas exactement non plus un éloge du genre?

The detective story was an object of fascination for the New Novelists. The list of their works appropriating crime fiction themes, characters or narrative structure is a long one, and includes texts by Robbe-Grillet, Simon, Pinget and Butor. The detective story's fixed form, and its principal theme of restoring order through reason and truth, proved irresistible to a literary movement aiming to question all aesthetic or epistemological assumptions behind novelistic fiction. How might we characterize this relationship of imitation between the New Novel and the detective story, which does not seem a hostile parody, but is not exactly a celebration of the genre either?

\section{INDEX}

Keywords : New Novel, crime fiction, detective story, Robbe-Grillet, Butor, Pinget

Mots-clés : Nouveau Roman, roman policier, polar, Robbe-Grillet, Butor, Pinget

\section{AUTEUR}

\section{SIMON KEMP}

Somerville College, University of Oxford 